\section{Rapid operant training of guinea pigs with lettuce as the appetitive reinforcer}

\author{
DAVID B. HARDER \\ Department of Psychology \\ Florida State University, Tallahassee, Florida 32306 \\ O. GREG BROCK \\ Department of Biological Science \\ Florida State University, Tallahassee, Florida 32306
}

and

\section{MICHAEL E. RASHOTTE \\ Department of Psychology \\ Florida State University, Tallahassee, Florida 32306}

The traditional view of the guinea pig as difficult to train (e.g., Roberts, 1972) has been altered by demonstrations that this species is adept in operant training when correct combinations of deprivation, training environment, and reinforcer are used (Berryman, 1976; Gundy, 1959; Jonson, Lyle, Edwards, \& Penny, 1975; Petersen, Prosen, Moody, \& Stebbins, 1977; Valenstein, 1959; Walloch \& Taylor-Spikes, 1976). Preliminary to employing the conditioned suppression procedure with guinea pigs, we sought a reliable and quick method for establishing a stable baseline of appetitively reinforced leverpressing. Valenstein (1959) demonstrated conditioned suppression in deprived guinea pigs, but the deprivation and training procedures for establishing the operant baseline in that experiment, and in most of the others cited above, were sufficiently complex and time consuming that we sought a simplified procedure. Failing to confirm a report that carrot juice is an effective reinforcer for nondeprived guinea pigs (Gundy, 1959), we subsequently encountered difficulty in achieving stable responding when plain or sweetened water was used as a reinforcer for water-deprived guinea pigs. This impasse was resolved when we utilized the well-known fact that guinea pigs have a strong affinity for fresh lettuce (e.g., Miller \& Murray, 1966; Roberts, 1972; Valenstein, 1959). The present paper describes the conditions under which we have found lettuce particularly effective as a reinforcer for establishing leverpressing in guinea pigs not deprived of food or water.

Five adult guinea pigs (Cavia porcellus) purchased from local pet suppliers were individually housed in a temperature/humidity controlled room with a 12-h light/ 12-h dark cycle. Purina guinea pig chow and water supplemented with vitamins (Avitron) were available con-

This project was supported in part by NIMH Training Grant MH-11218 and NIH Grant NS-11668. Requests for reprints should be addressed to Michael E. Rashotte, Department of Psychology, Florida State University, Tallahassee, Florida 32306 . tinuously. The animals had intermittent access to lettuce in their cages before and after they arrived at the laboratory. The guinea pigs were trained in a standard operant conditioning chamber for rats, equipped with a thin $(.5 \mathrm{~cm})$ metal lever $2.7 \mathrm{~cm}$ wide which projected $2.2 \mathrm{~cm}$ into the chamber. The lever was mounted $3.2 \mathrm{~cm}$ above the grid floor, to the left of center on the intelligence panel. The chamber was constantly illuminated by a masked 15-W houselight. Each leverpress produced a brief feedback flash from a cue light located $5.3 \mathrm{~cm}$ above the lever, while a reinforced press also produced an auditory feedback click which was immediately followed by lettuce reinforcement. The lettuce was manually inserted through a hole $12.7 \mathrm{~cm}$ to the right of the lever and $8.5 \mathrm{~cm}$ above the floor. The guinea pigs readily consumed as many as 120 approximately $2 \times 3 \mathrm{~cm}$ hand-torn pieces of lettuce in $55 \mathrm{~min}$ without signs of satiation. The size of the pieces was not especially critical, except that larger pieces required a longer consummatory time.

Magazine training was usually accomplished within $15 \mathrm{~min}$ following a short (5- to $10-\mathrm{min}$ ) exploration period in the first training session. A feedback click signaling reinforcement was presented before each insertion of lettuce. If the animal did not readily eat, the lettuce was gently shaken while protruding from the hole in the intelligence panel to attract the animal to it.

The subjects were then "shaped" to press the lever with no specific response topography required. The dimensions of the lever may be relevant since leverpressing was principally by biting. This action occurred spontaneously during exploration and seems related to the natural response topographies successfully conditioned in previous studies (Berryman, 1976; Jonson et al., 1975; Petersen et al., 1977; cf. Valenstein, 1959). Only one animal pressed the lever with its paw, and then only in isolated instances after pressing was well established (see Valenstein, 1959).

Four animals began responding on a continuous reinforcement (CRF) schedule within two 25-min sessions, while a fifth animal was shaped to touch the lever in the first session but did not reliably make the full response until Session 10. Once the animals were responding well on CRF, successively longer fixed-ratio (FR) schedules were introduced until the subjects responded reliably on FR 15. FR training required from 5 to 7 sessions. A representative cumulative record of responding on FR 15 is shown in the upper panel of Figure 1. For two animals the schedule subsequently was changed to variable interval (VI) $30 \mathrm{sec}$ and one of the animals continued on that schedule. After four sessions of VI $30 \mathrm{sec}$ the other animal was trained on VI $45 \mathrm{sec}$ for seven sessions and then was placed on VI $60 \mathrm{sec}$. The VI schedules were generated according to the Fleshler-Hoffman (1962) formula. The bottom panel 


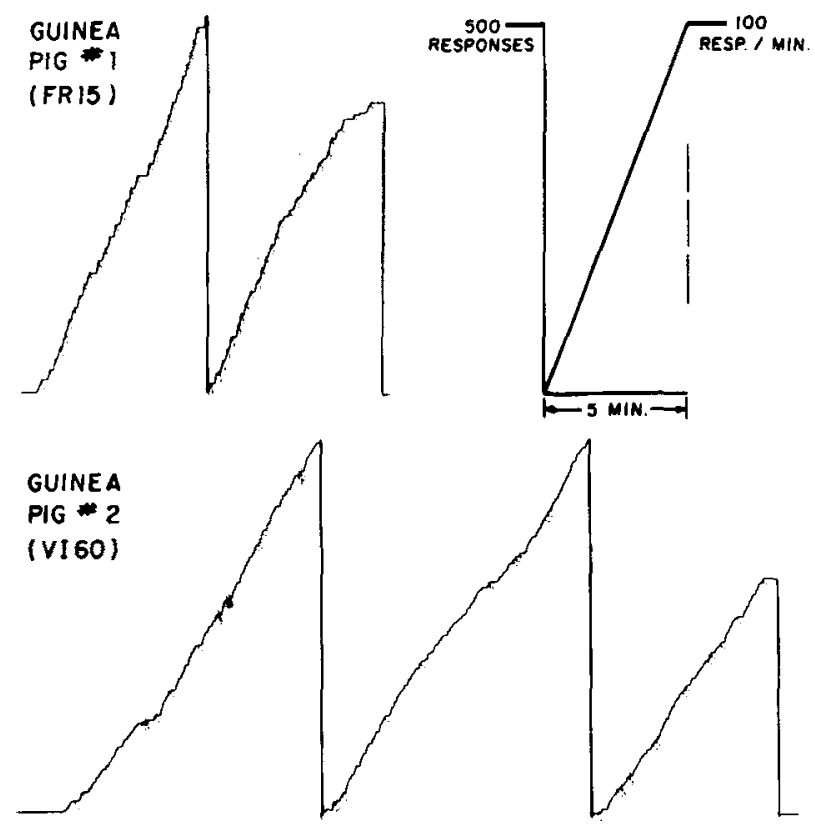

Figure 1. Cumulative records of leverpressing by nondeprived guinea pigs for lettuce reinforcer. The upper panel shows responding on FR 15 on the 10th day from the beginning of training. The bottom panel shows responding after 6 days on VI 60-sec. A downward deflection of the pen indicates reinforcement. The recorder continued to run during reinforcement periods, so a part of the pausing after reinforcement in these records results from time spent eating.

of Figure 1 illustrates the performance of Subject 2 in Session 6 on VI $60 \mathrm{sec}$. The data in Figure 1 show that lettuce is an effective reinforcer for establishing schedule control of leverpressing in nondeprived guinea pigs.

With the VI baseline established, some preliminary information on conditioned suppression was obtained from two animals. In this part of the project the cue light over the lever no longer provided response feedback but was lighted continuously. Removing the feedback cue produced no effect on responding. The CS was a 20-sec darkening of the houselight. Leverpressing was partially disrupted during the CS when it was first presented alone. In the next four sessions each CS terminated with an overlapping 1-mA scrambled electric shock presented through the grid floor. Shock duration of $.5 \mathrm{sec}$ had little observable effect on the animals' behavior. Leverpressing was briefly disrupted by the shock when duration was increased to $1.0 \mathrm{sec}$ and some suppression during the CS was evident after three to five trials. When shock duration was increased to $2.0 \mathrm{sec}$, suppression in the CS was complete after only one or two CS-US pairings (cf. Valenstein, 1959) and longer periods of immobility followed shocks. Four or five CS-US trials were given in each session, with an average intertrial interval of $10 \mathrm{~min}$.

Using lettuce as a reinforcer, we rapidly established stable appetitively reinforced leverpressing in the guinea pig. without relatively demanding deprivation and training procedures (e.g., Jonson et al., 1975). Long periods of deprivation and acclimation to the apparatus were not necessary. In fact, four of the five subjects were used within $48 \mathrm{~h}$ of receipt in the lab, and all subjects ate readily in the test chamber during the first training session. Once the leverpress response was shaped, progress on more demanding schedules was relatively rapid. The lettuce reinforcer was adequate to maintain steady responding on VI $60 \mathrm{sec}$ for at least 55-min sessions, with every indication that both the schedule and the session length could be increased. The VI baseline became increasingly stable as training continued. In the initial sessions responding was characterized by bursts of biting separated by pauses. In later sessions bursts were replaced by more steady responding on the lever. While the performance shown in Figure 1 is somewhat variable as a baseline for conditioned suppression, we expect that the baseline would become more suitable with extended training.

Lettuce may be an unusually effective reinforcer for the guinea pig because it is similar to foods in that species' natural diet. Other plants may prove equally effective. In future work it will be desirable to automate presentations and standardize portions of this type of reinforcer. One solution to this problem is to employ a universal feeder such as the DSI UF-100 manufactured by Davis Scientific Instruments ${ }^{1}$ to dispense portions of fixed size.

\section{REFERENCES}

Berryman, J. C. Operant conditioning in non-deprived adult and infant guinea pigs. Journal of the Experimental Analysis of Behavior, 1976, 25, 400.

Fleshler, M., \& Hoffman, H. S. A progression for generating variable interval schedules. Journal of the Experimental A nalysis of Behavior, 1962, 5, 529-530.

Gundy, R. F. Some techniques in operant conditioning of the guinea pig. Joumal of the Experimental Analysis of Behavior, $1959,2,86$.

Jonson, K. M., Lyle, J. G., EDwards, M. J., \& Penny, R. H. C. Problems in behavioural research with the guinea pig: A selective review. Animal Behaviour, 1975, 23, 623-639.

Miller, J. C., \& Murray, F. S. Guinea pig's immobility response to sound: Threshold and habituation. Journal of Comparative and Physiological Psychology, 1966, 61, 227-233.

Petersen, M. R., Prosen, C. A., Moody, D. B.. \& Stebins, W. C. Operant conditioning in the guinea pig. Journal of the Experimental Analysis of Behavior, 1977, 27, 529-532.

RoBerTs, M. F. Guinea pigs for beginners. Neptune City, N.J: T. F. H. Publications, 1972.

VALEnStein, E. S. The effect of reserpine on the conditioned emotional response in the guinea pig. Journal of the Experimental A nalysis of Behavior, 1959, 2, 219-225.

Walloch, R. A., \& TAYLoR-Spikes, M. Auditory thresholds in the guinea pig: A preliminary report of a behavioral technique employing a food reward. The Laryngoscope, 1976, 86, 1699-1705.

\section{NOTE}

1. Davis Scientific Instruments, 12137 Cantura Street, Studio City, California 91604.

(Received for publication September 23, 1977; revision accepted October $28,1977$. ) 